acceptable mortality risk. In the elective setting, shared decision making via MDT is commonplace, appropriate patients are selected, acute complications are rare, and long-term outcomes are favourable with low in-stent restenosis rates. The acute setting, by default associated with a more adverse prognosis, presents more challenge - opportunity for MDT discussion is limited and patient/procedural factors can reduce intracoronary imaging rates. Procedural outcomes are favourable but all-cause mortality in LMS STEMI patients is predictably high. Finally, shared decision making is of paramount importance in this uncertain area and a further update to the guidelines will be welcomed.

Conflict of Interest none

\section{A SURVEY OF CURRENT LEARNING METHODS USED BY INTERVENTIONAL CARDIOLOGY TRAINEES IN THE UNITED KINGDOM}

Anenta Ramakrishnan. Imperial College London, London, UK

10.1136/heartjnl-2021-BCS.66

Introduction Interventional Cardiology is a specialism using minimally invasive catheter-based interventions for cardiac treatment, and therefore is largely procedure-based. Trainees enter sub-specialty training at beginner or intermediate level, and by completion of two years of sub-specialty training they are expected to be expert and independent in basic coronary angiography, simple angioplasty and stenting, pericardiocentesis and to be intermediate level in more complex procedures such as revascularisation of complete total occlusions. Sub-specialty training is usually a two- year programme based at one or more training hospitals. There is a curriculum and set of competencies which must be achieved, and this is assessed continually throughout the year and appraised annually (GMC, 2018). AimThe aim of this research was to understand the learning methods experienced by Interventional Cardiology trainees in the United Kingdom and explore the value of these methods as perceived by trainees.

Methods Taking a pragmatist learning approach within the framework of critical theory, a quantitative survey was conducted. Trainees were approached via social media networks, local mailing lists and directly via Training Programme Directors where possible. The survey was delivered and analysed through Google Forms. Ethical approval was obtained from the University of Dundee.

Results Thirty-four interventional trainees (ST5 level and above) in the United Kingdom responded to the survey. Catheter laboratory training sessions and case based discussions were considered the most useful learning methods. Over half of respondants reported that their learning would be enhanced if they had greater opportunity to utilise catheter laboratory training sessions, case-based discussion and simulation. Over one third are planning or have completed an out of training fellowship abroad, and nearly a third are planning of have completed an out of training fellowship in the UK or abroad. Nearly a half of respondants reported they would like to training coronary, structural and complex coronary internvetion, whilst $27 \%$ said they would like to train in coronary and structural intervention, and $29 \%$ said they would like to training in coronary and complex coronary intervention. Over one third of respondants said they would additional further training in other sub-specialties (most commonly devices and imaging).

Conclusion We report our findings on learning methods utilised by Interventional Cardiology trainees and in the UK, as well as findings on trainee preferences for the future direction of training.

Conflict of Interest None

\section{YOUNG PATIENTS WITH NON-ST ELEVATION ACUTE CORONARY SYNDROMES: BASELINE CHARACTERISTICS, IN-HOSPITAL MANAGEMENT AND OUTCOMES}

Zainab Dakhil. Ibn Al Bitar Cardiac Centre, Al-kindy College Of Medicine/university of Baghdad, Baghdad, Iraq

\subsection{6/heartjnl-2021-BCS.67}

Background There is an alarming increase in prevalence of acute coronary syndromes in young population, yet, there is limited data about the in-hospital management strategy and outcomes in this population. Furthermore, up to our knowledge, there is no data from Iraq regarding clinical profile and in-hospital management of young patients with NSTEACS.

Purpose We aimed to assess the clinical profile, in-hospital management and outcomes of hospitalized young patients whose age $\leq 50$ years who were presented with NSTEACS.

Methods This study included admitted patients with NSTEACS whose age $\leq 50$ years, baseline characteristics and inhospital management as well as outcomes were recorded.

Results Total n. $=280,80$ patients were excluded from analysis due to missing some of data, $23 \%$ of included patients were at age $\leq 50$ years, among whom $84.8 \%$ were males vs $15.2 \%$ females. Mean age of young patients was $43.6 \pm 6.3$ years. Of this young patient population: hypertension, diabetes and prior history of IHD reported in 52.2\%, 43.5\% and $32.6 \%$ respectively while smoking, hyperlipidemia and positive family history of IHD were reported in 56.5\%, 19.6\% and $34.8 \%$ respectively. Dyspnea at presentation was reported in $19.6 \%$, troponin was positive in $43.5 \%$, mean urea was $31.6 \pm 16 \mathrm{mg} / \mathrm{dl}$, mean creatinine was $0.86 \pm 0.33$ $\mathrm{mg} / \mathrm{dl}$, mean $\mathrm{Hb}$ was $14.2 \pm 1.7 \mathrm{gm} / \mathrm{L}$, their mean left ventricular EF\% was $57.4 \pm 10.4$. Young patients were referred for catheterization in $84.8 \%$, however, at high GRACE risk class; they were treated invasively less than young patients at low

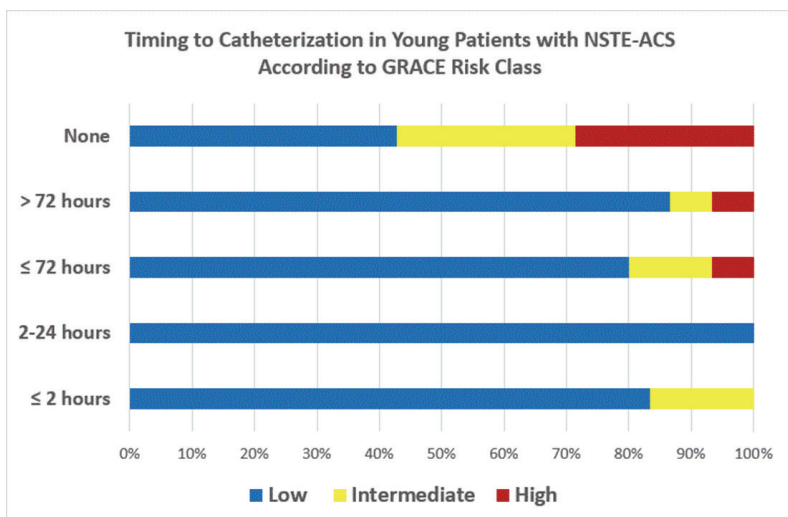

Abstract 67 Figure 1 